\title{
Cardiomiopatía amiloídea por transtiretina: diagnóstico y manejo en la actualidad
}

\author{
Felipe Castañía ${ }^{1}$, Teresa Massardo ${ }^{2}$, Jaime Álvarez ${ }^{3}$, Jorge Jalil ${ }^{1}$, Douglas Greig ${ }^{1}$, Paul Mcnab $^{1}$, \\ Marcelo Llancaqueo ${ }^{4}$, Rodrigo Jaimovich ${ }^{5}$. \\ 1. División de Enfermedades Cardiovasculares, Pontificia Universidad Católica de Chile. \\ 2. Sección Medicina Nuclear, Departamento de Medicina, Hospital Clínico Universidad de Chile. \\ 3. Departamento de Medicina, sección de Cardiología, Hospital Salvador \\ 4. Departamento Cardiovascular, Hospital Clínico Universidad de Chile. Cardiología, Clínica Las Condes. \\ 5. Medicina Nuclear, Clínica Las Condes.
}

Conflictos de interés. Los Doctores Massardo, Álvarez y Jalil conducen el Registro de Amiloidosis Cardíaca en Santiago

(Sochicar, Laboratorio Pfizer) y han recibido honorarios por charlas en relación con el diagnóstico de Amiloidosis (Laboratorio Pfizer).

La cardiomiopatía amiloide por transtiretina (CATTR) es una enfermedad caracterizada por depósito extracelular de fibrillas amiloides en el miocardio, a partir de transtiretina mal plegada, generando una miocardiopatía restrictiva. Esta proteína mal plegada puede tener origen hereditario o adquirido, siendo más frecuente en adultos mayores. La CA-TTR ha surgido como una causa subdiagnosticada de insuficiencia cardíaca con fracción de eyección preservada (IC FEp). El pilar fundamental para su diagnóstico es la alta sospecha clínica, basada en diversas banderas de alerta ya que la sintomatología que provoca suele ser inespecífica. Como veremos en esta revisión, el diagnóstico puede sustentarse con la cintigrafía ósea, reservando para situaciones particulares la toma de biopsia. Con el advenimiento de nuevas terapias que impactan en la sobrevida de esta enfermedad, el tiempo para realizar el diagnóstico certero y la diferenciación de otras causas de amiloidosis cardíaca como la de cadenas livianas, se ha tornado crucial.

Palabras clave: Amiloidosis, cardíaca; cardiomiopatía amiloidea; transtiretina.

Correspondencia: 


\section{Transthyretin amyloid cardiomyopathy: a review.}

Transthyretin amyloid cardiomyopathy (ATTR-CM) is a disease characterized by extracellular deposition of amyloid fibrils in the myocardium, from misfolded transthyretin, generating a restrictive cardiomyopathy. This misfolded protein may be inherited or acquired, and is more prevalent in elderly patients. ATTR-CM has emerged as an underdiagnosed cause of heart failure with preserved ejection fraction (HF-PEF). The fundamental pillar for its diagnosis is high clinical suspicion since the symptoms are usually nonspecific. The diagnosis can be made from bone scintigraphy, reserving myocardial biopsy for particular situations. With the advent of new therapies that affect the survival of these patients, a timely diagnosis has become crucial.

Keywords: cardiac amyloidosis; transthyretin amyloid cardiomyopathy 


\section{Introducción:}

La cardiomiopatía amiloide o amiloídea (CA) por transtiretina, es un tipo de amiloidosis sistémica, con infiltración y depósito de agregados fibrilares insolubles de transtiretina (TTR) a nivel extracelular de los cardiomiocitos, generando alteraciones cardíacas estructurales y funcionales. ${ }^{1}$ Se clasifica según la presencia o ausencia de mutación genética de TTR, en la forma hereditaria (ATTRh) o natural (wild-type: ATTR-wt). ${ }^{2}$ Es una causa de insuficiencia cardíaca de diagnóstico complejo en adultos mayores ${ }^{3}$ y forma parte de las causas infiltrativas de la miocardiopatía restrictiva ${ }^{4}$ y del espectro de la falla cardíaca con fracción de eyección preservada (IC FEp). Reportes recientes sugieren que el subtipo ATTR-wt tiene una prevalencia de hasta $13 \%$ como causa de IC FEp en pacientes mayores de 60 años ${ }^{5}$ y $16 \%$ en pacientes con estenosis aórtica de bajo flujo mayores de 80.6

Tradicionalmente, el subdiagnóstico de CA se ha atribuido a la heterogeneidad en su forma de presentación, a la percepción de enfermedad poco frecuente o rara, a la necesidad de derivación a recintos asistenciales con capacidad de confirmación histológica y, hasta poco tiempo atrás, a la ausencia de tratamientos eficaces.

Sin embargo, gracias al avance tanto en métodos diagnósticos no invasivos, como de las imágenes cardíacas incluyendo las isotópicas y las nuevas terapias farmacológicas que estabilizan o suprimen la producción de TTR, se ha logrado impactar en el pronóstico en esta enfermedad. En consecuencia, se hace imprescindible cambiar el enfoque clínico en cuanto a diagnóstico y tratamiento precoz. En el presente artículo se revisan los conceptos actualizados en relación con los mecanismos de CA-TTR, cuadro clínico, métodos y algoritmos de diagnóstico y tratamiento.

\section{Fisiopatología}

La TTR, previamente denominada "prealbúmina", es una proteína plasmática sintetizada en el hígado (y menos del $5 \%$ en plexos coroideos y epitelio pigmentario retinal). TTR posee una configuración tetramérica y transporta tiroxina y proteínas que se unen a retinol (de ahí el nombre transtiretina). Esta proteína tiene la capacidad de disgregarse en dímeros y monómeros, que pueden agregarse y formar fibras amiloides insolubles ${ }^{7}$ que se depositan en el corazón y otros tejidos.

La CA-TTR, se clasifica en 2 subgrupos, según la presencia o ausencia de mutación genética de TTR:

\section{A) Amiloidosis por transtiretina hereditaria (ATTR-h):} La forma hereditaria de ATTR, secundaria a mutaciones genéticas de TTR que aumentan la probabilidad de disociación de tetrámeros a monómeros de TTR con baja estabilidad conformacional y alta tendencia a agregación en fibrillas amiloides, que pueden depositarse en corazón, nervios u otros tejidos. Existen más de 120 mutaciones de TTR, y hay una correlación genotipo-fenotipo que determina un compromiso cardíaco o neurológico ${ }^{8}$. A pesar de ser una enfermedad rara, es endémica en algunas zonas geográficas y existen mutaciones específicas que generan compromiso fundamentalmente

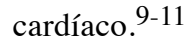

Las manifestaciones clínicas de la enfermedad hereditaria son similares a las de la ATTR-wt, con mayor compromiso neurológico en la ATTR-h, con polineuropatía sensitivo-motora simétrica y ascendente. Existen también distintos grados de disfunción autonómica, como hipotensión ortostática, incontinencia urinaria, disfunción eréctil y síncope ortostático.

\section{B) Amiloidosis por transtiretina "wild-type" o natu- ral (ATTR-wt):}

Tradicionalmente denominada CA senil, en relación con la edad tardía de inicio ( $>80$ años) aunque puede tener un comienzo más precoz, incluso a los 60 años. Corresponde probablemente a la forma más frecuente de CA (excluyendo a la CA por cadenas livianas y a las de origen inflamatorio) de entre las más de 30 proteínas que pueden formar fibrillas de amiloide y depositarse. Se desconoce el motivo por el cual TTR se torna inestable y favorece su agregación, pero aparentemente se relacionaría con la edad.

El depósito de amiloide en la amiloidosis TTR-wt puede ocurrir también a nivel extracardíaco, generando característicamente: síndrome del túnel carpiano (particularmente bilateral), que suele preceder en 5 a 10 años a las manifestaciones cardíacas $^{12}$, rotura espontánea distal del tendón del bíceps ${ }^{13}$ y estenosis espinal lumbar o raquiestenosis. ${ }^{14}$ En la ATTR-wt es infrecuente el compromiso neurológico (periférico y/o autonómico), alcanzando hasta el $10 \%$ de los pacientes. ${ }^{15}$

\section{Presentación clínica de la amiloidosis cardíaca}

La forma clásica de presentación de pacientes con CA suele ser la IC FEp, reflejando el desarrollo de disfunción diastólica secundaria a infiltración amiloidea e intolerancia al ejercicio debido a un gasto cardíaco bajo. Sin embargo, en hasta $1 / 3$ de los pacientes se pesquisa 
en etapas tardías, como insuficiencia cardíaca con fracción de eyección reducida (IC FEr). No es infrecuente que los pacientes presenten síncope en relación con el ejercicio, debido a un volumen eyectivo relativamente fijo. ${ }^{16}$ Además, suele acompañarse de hipotensión arterial, que complica el manejo de la falla cardíaca. CA-TTR puede estar presente en hasta el $16 \%$ de los pacientes con estenosis aórtica calcificada moderada a severa $^{17}$, y se asocia a un incremento del riesgo de insuficiencia cardíaca, mayor mortalidad y a falta de respuesta clínica al reemplazo valvular aórtico. ${ }^{18}$

\section{Métodos diagnósticos no invasivos}

Biomarcadores cardíacos. Habitualmente los valores de troponina y de NT-ProBNP se encuentran leve o moderadamente elevados en forma crónica en estos pacientes lo que podría relacionarse con el grado de infiltración miocárdica por el amiloide. Estos biomarcadores tienen utilidad como factor pronóstico, permitiendo la estratificación del riesgo y el pronóstico en la CA-TTR en distintos estadios clínicos. ${ }^{19}$

Electrocardiograma. Cumple un importante rol en la sospecha diagnóstica de CA-TTR. Los hallazgos electrocardiográficos más comunes son el patrón de pseudoinfarto (QS en 2 derivadas contiguas) ${ }^{20}$ en hasta el $50 \%$ de los pacientes, simulando un infarto al miocardio previo. El 25-40\% de los pacientes puede tener criterios de bajo voltaje (amplitud del QRS $<0.5 \mathrm{mV}$ en todas las derivadas de las extremidades o $<1 \mathrm{mV}$ en todas las derivadas precordiales) ${ }^{21}$, lo que asociado a engrosamiento de la pared del ventrículo izquierdo (VI) en el ecocardiograma da el sello clásico de esta enfermedad. Esto se explica porque el engrosamiento ventricular ocurre por infiltración miocárdica más que por hipertrofia de los cardiomiocitos. Sin embargo, alrededor de un $25 \%$ de los pacientes con CA-TTR cumple criterios de voltaje para hipertrofia ventricular izquierda. ${ }^{22}$

También se produce infiltración de fibrillas amiloides en las aurículas y/o sistema de conducción, con una alta prevalencia de arritmias supraventriculares, principalmente fibrilación auricular (en hasta el 70\% de los pacientes con ATTR-wt) y/o bloqueos AV, que pueden presentarse incluso años antes de la aparición de la insuficiencia cardíaca. $^{23}$

Ecocardiograma. Esta herramienta clínica ampliamente disponible puede entregar las primeras pistas de presencia de CA-TTR, dado que el depósito de amiloide infiltra todas las cámaras cardíacas (predominantemente ventriculares) y las válvulas. La fracción de eyección del VI está clásicamente preservada en los estadios precoces y la disfunción diastólica progresa a medida que continúa el depósito de fibrillas amiloídeas en el miocardio. Los hallazgos ecocardiográficos más típicos, incluyen: engrosamiento de las paredes ventriculares, generalmente asimétrico en CA-TTR, exhibiendo el máximo de hipertrofia en el septum (>12 mm), cavidades ventriculares no dilatadas o pequeñas, engrosamiento valvular, dilatación auricular, derrame pericárdico y aspecto granular del miocardio (Figura 1). El estudio de la deformación miocárdica longitudinal por Speckle Tracking constituye un importante avance en el estudio de CA, otorgando mayor sensibilidad y especificidad al diagnóstico y al pronóstico (su utilización tiene recomendación clase I y nivel de evidencia B) ${ }^{24} \mathrm{El}$ depósito predominante de amiloide en el intersticio celular en los segmentos basales y medios del miocardio respetando los segmentos apicales, genera una imagen de menor deformación en los segmentos más comprometidos, lo que se conoce como el signo de la "bandera japonesa" o "cherry on top" con relación apical/basal $>2: 1$ (Figura 2). ${ }^{25}$ Otra aproximación ecocardiográfica está determinada por el compromiso precoz de la deformación longitudinal, antes del deterioro de la fracción de eyección, con una relación FE/SGL mayor a 4, especialmente útil en pacientes con grosores miocárdicos menores a $16 \mathrm{~mm}$ y FEVI mayor a $55 \% .^{26}$

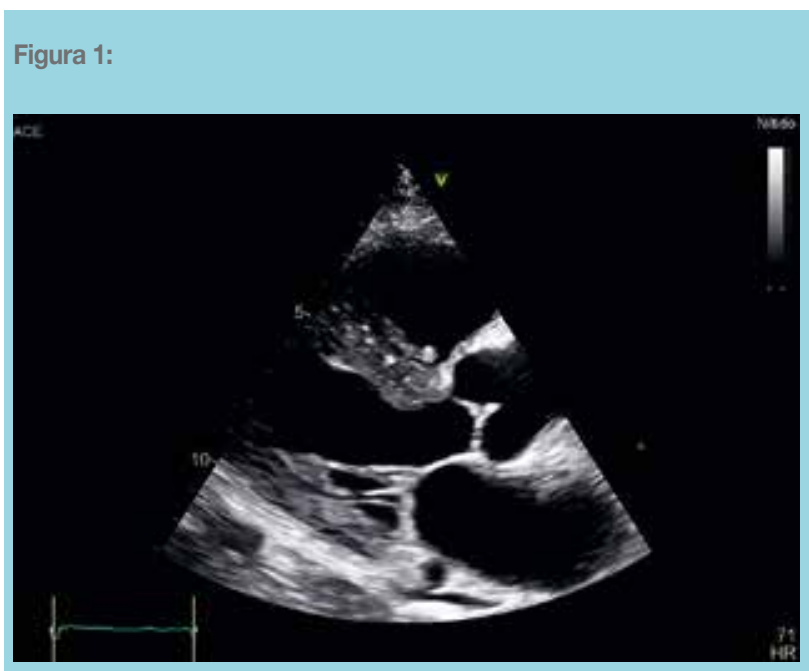

Figura 1: Hipertrofia ventricular izquierda y aspecto granular ecogénico de la pared del VI, sobre todo en el septum interventricular en paciente con amiloidosis cardíaca AL (ECO 2D, eje paraestenal largo). 


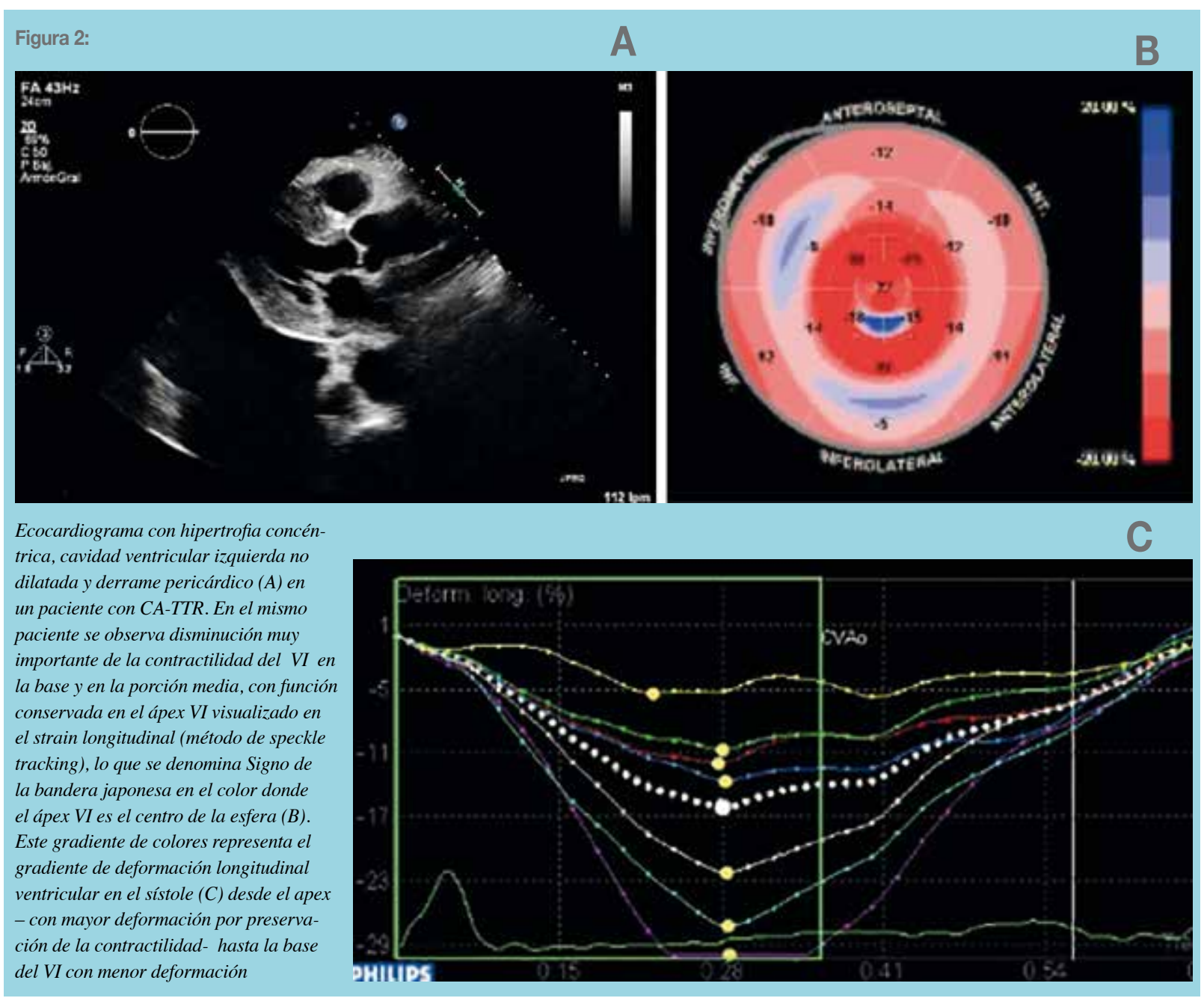

Resonancia nuclear magnética (RNM) cardíaca (Figura 3). La RNM cardíaca entrega información precisa sobre la estructura y funcionalidad del corazón, con ciertas ventajas sobre la ecocardiografía. Es útil para diferenciar amiloidosis de enfermedades no amiloídeas ${ }^{27}$ al caracterizar el tejido miocárdico en base a sus propiedades magnéticas intrínsecas, que se pueden acentuar con medios de contraste en base a gadolinio (un medio de contraste extracelular que no ingresa a los cardiomiocitos a menos que exista daño de la membrana celular). Sin embargo, la RNM es incapaz de diferenciar CA-ATTR de cardiomiopatía amiloídea por cadenas livianas (CA-AL). ${ }^{28}$

En la CA-TTR hay 3 tipos de patrones al evaluar el realce tardío con gadolinio (LGE): Ausente, subendocárdico difuso (en etapas tempranas) o transmural (al progresar la enfermedad). Adicionalmente, al aumentar el tejido extracelular, como en la CA, aumentará la concentración de gadolinio en este lugar.
Secuencias Paramétricas. En los últimos años ha cobrado importancia la cuantificación de las propiedades del miocardio mediante la medición de los tiempos de relajación longitudinal (T1 o spin-lattice relaxation) y transversal (T2 o spin-spin relaxation) ${ }^{29}$ Los valores de normalidad, especialmente de $\mathrm{T} 1$, dependen de la intensidad del campo magnético de cada equipo, de las secuencias de pulso utilizadas (MOLLI, ShMOLLI) y del momento de adquisición dentro de ciclo cardíaco (diástole versus sístole), siendo necesaria la estandarización de valores de normalidad en cada centro.

Mapeo T1. El tiempo de relajación longitudinal aumenta notablemente en presencia de fibrosis intersticial siendo el caso más extremo la CA. Su valor normal aproximado es $960 \pm 30 \mathrm{~ms}$ (equipo de 1,5 Teslas). Recientemente se han descrito que valores sobre $1164 \mathrm{~ms}$ tienen un valor predictivo positivo de $98 \%$, mientras que valores menores a $1036 \mathrm{~ms}$ tienen un valor predicti- 


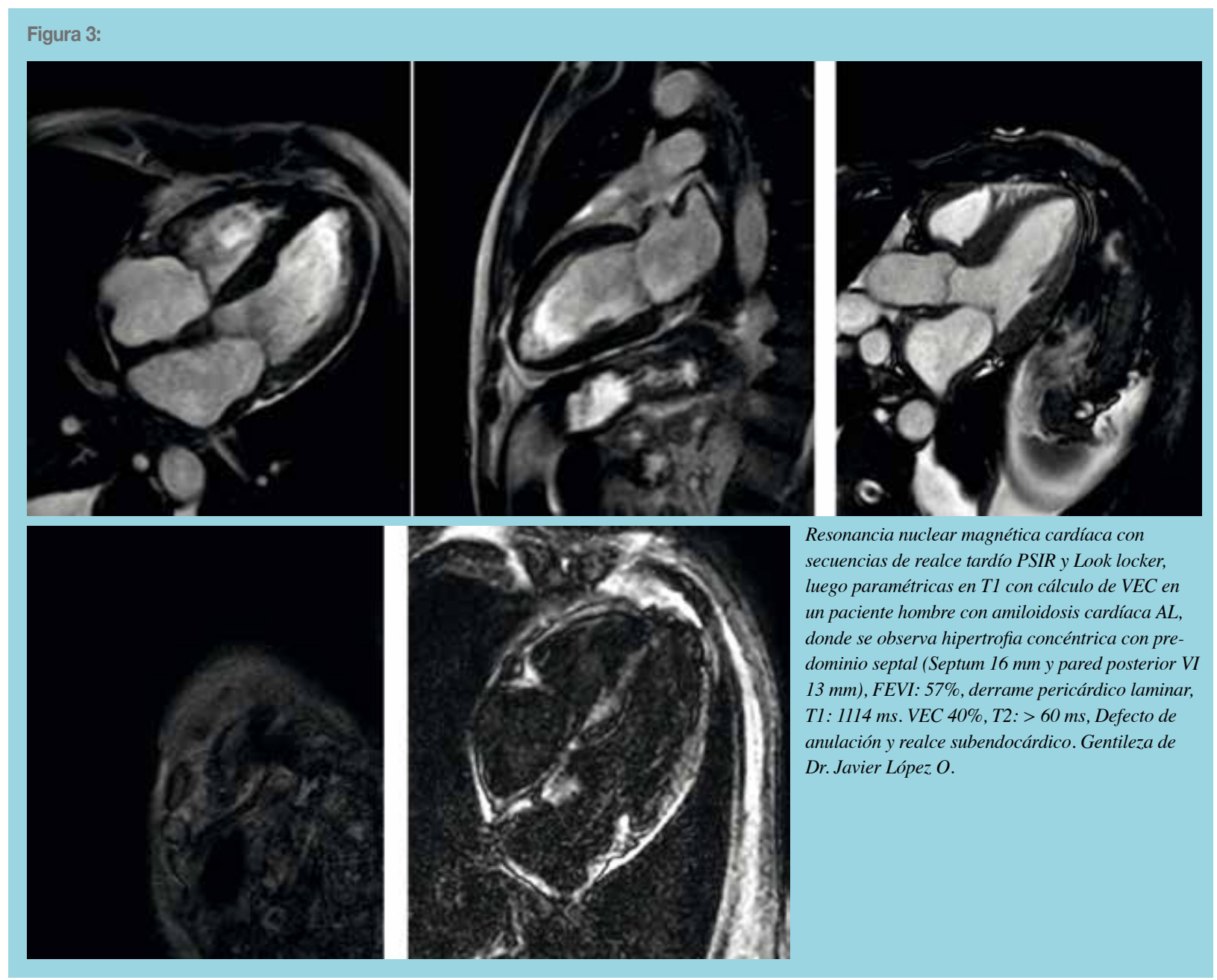

vo negativo de $98 \%{ }^{30}$ Esto es relevante en CA porque permite hacer diagnóstico sin gadolinio en pacientes con insuficiencia renal.

Otro elemento muy importante que permite realizar diagnóstico y seguimiento de los pacientes es el cálculo del Volumen Extra Celular (VEC), derivado de la relación entre T1 nativo (precontraste), T1 post-contraste de gadolinio y el hematocrito, con un valor normal de 25\%. Los pacientes con CA, especialmente CA-TTR, presentan un gran aumento de volumen extracelular (VEC) secundario al depósito de material amiloídeo (valores mayores de 40\%). El riesgo relativo de mortalidad aumenta en 1,2 por cada $59 \mathrm{~ms}$ de aumento de $\mathrm{T} 1$ y de $3 \%$ de aumento en el VEC.

Mapeo T2. El tiempo de relajación transversal aumenta en presencia de edema. En la CA-AL se puede observar un valor de T2 elevado por efecto citotóxico inducido por el depósito de cadenas livianas, pero no existe evi- dencia que permita utilizar esta información para diferenciar CA-TTR de CA-AL.

Cintigrafía con bifosfonatos. Su utilidad radica como método diagnóstico no invasivo de CA-TTR. El mecanismo por el cual ciertos trazadores óseos, como pirofosfato (PYP), marcados con Tecnecio 99 se acumulan en esta patología, es desconocido. Con la escala visual de Perugini (semi-cuantitativa), se compara el nivel de captación del trazador a nivel cardíaco con el de las costillas, clasificándolo en: grado 0 (ausencia de captación cardíaca y captación ósea normal), grado 1 (captación cardíaca inferior a la captación ósea), grado 2 (captación cardíaca equivalente a la captación ósea) y grado 3 (captación cardíaca mayor que la ósea, con poca o nada captación de costillas (Figura 4). ${ }^{31}$

En el método de análisis cuantitativo se calcula la relación entre el la captación del radiotrazador a nivel cardíaco con la región pulmonar contralateral (H/CL). Los 


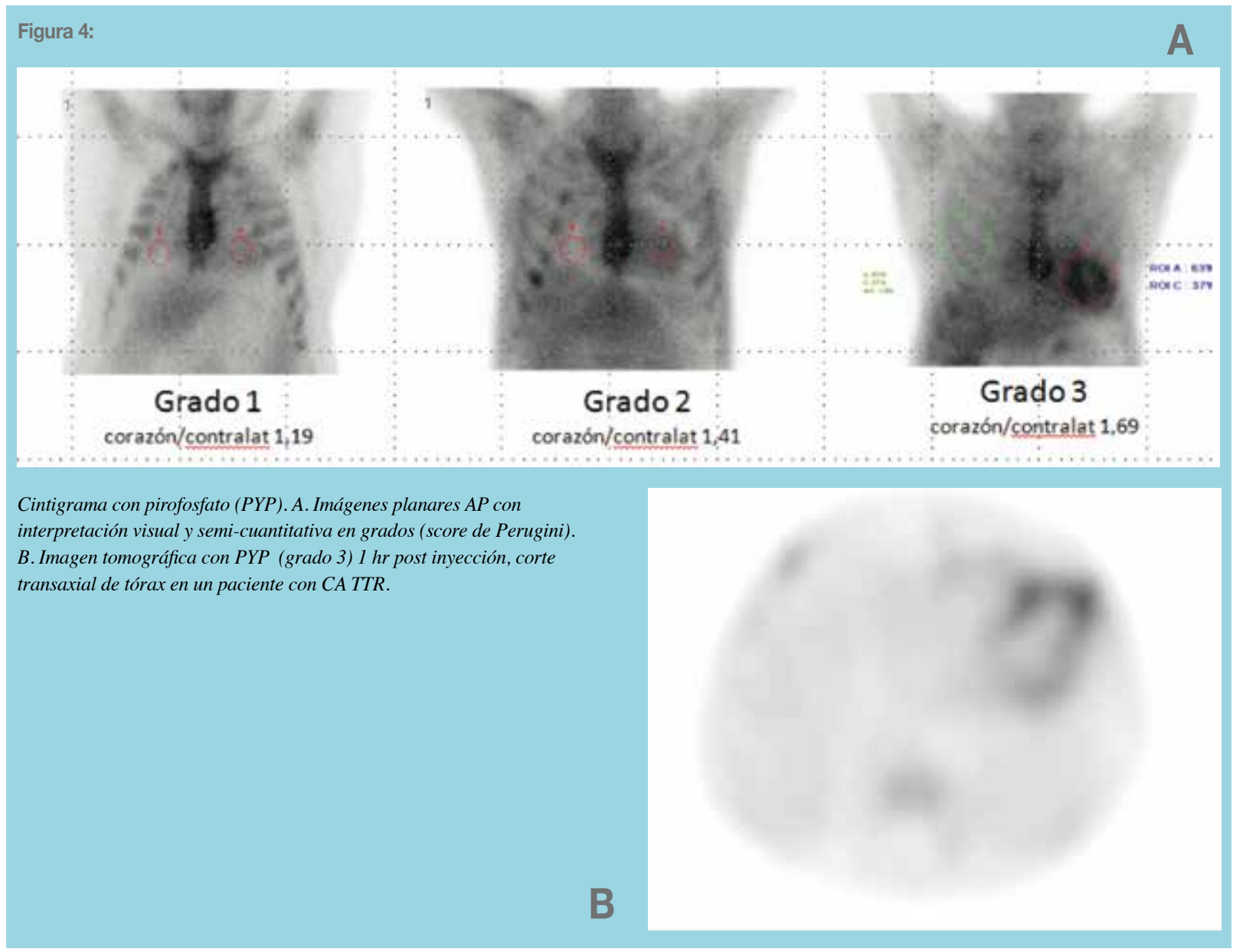

grados 2 o 3 en el método semi-cuantitativo (Perugini) o una relación $\mathrm{H} / \mathrm{CL}>1.5$ en el método cuantitativo, en ausencia de proteínas monoclonales en sangre y orina, son diagnósticos de esta enfermedad (especificidad cercana al $100 \%) .32$

Los valores anteriores se aplican al adquirir imágenes planares y tomográficas o SPECT de tórax a 1 hora, ya que a las 3 horas el índice cuantitativo límite disminuye a 1.3. La captación cardíaca es difusa en miocardio y con las imágenes tomográficas se puede diferenciar claramente una fractura costal o derrame. La sensibilidad de la técnica es alta y captaciones de baja intensidad pueden observarse en $\mathrm{CA}-\mathrm{AL}$, en etapas precoces de CA-TTR y en algunas formas hereditarias.

Existen algunas dificultades en las siguientes condiciones: infarto del miocardio y fracturas costales previas o incluso derrame pleural que podrían interferir con la evaluación del trazador en miocardio con amiloidosis, especialmente si no se efectúa tomografía computarizada por emisión de fotón único (SPECT).
La especificidad de esta técnica se incrementa si se descarta la presencia de cadenas livianas (inmunofijación en sangre y orina), pues pacientes con CA-AL también pueden tener captación del PYP.

Existen otros marcadores fosfonados útiles con este mismo propósito (ácido difosfono-propanodicarboxílico, DPD) e hidroxi-metilendifosfonato, HMDP).

Se reconoce que realizando la técnica adecuadamente se la puede considerar como una alternativa segura y no invasiva de la biopsia cardíaca. ${ }^{33-34}$

\section{Métodos diagnósticos invasivos}

Biopsia endomiocárdica (BEM) y biopsia extracardíaca.

La BEM es el estándar en el diagnóstico de CA. En la CA-TTR permite documentar la infiltración amiloide al microscopio óptico con tinción de Rojo Congo, dando un color pardo rojizo (Figura 5), que bajo luz polarizada dará característicamente birrefringencia color verde manzana. Para identificar el tipo de precursor proteico mal plegado, se utiliza inmunohistoquímica o espectro- 


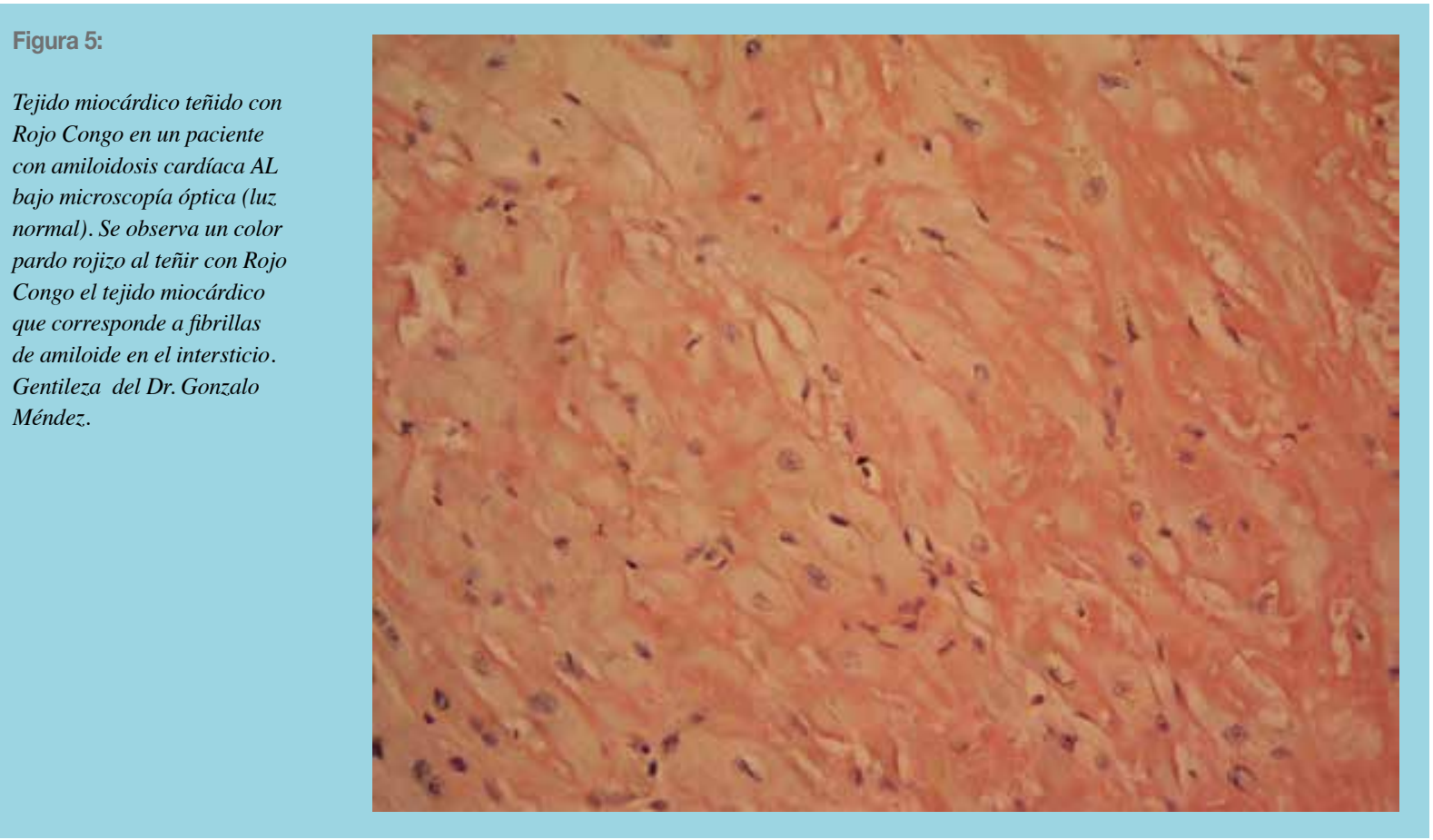

metría de masas (método de referencia). La BEM tiene un rol diagnóstico mucho más relevante en la $\mathrm{CA}-\mathrm{AL}$ por la menor sensibilidad y especificidad de la cintigrafía con PYP en esta condición. En la CA-TTR, donde la especificidad y sensibilidad de la cintigrafía con PYP u otro compuesto fosfonado son altas, la BEM se reserva solamente para 2 situaciones:

(1) Falta de disponibilidad de esta técnica.

(2) Cintigrafía ósea con 99mTc-PYP negativa y alta sospecha diagnóstica de CA-TTR.

La biopsia de otros tejidos (grasa abdominal subcutánea, piel, glándulas salivales o tracto gastrointestinal) tiene una sensibilidad variable en diagnóstico de CATTR, por lo que un resultado negativo en un órgano no afectado, no excluye el diagnóstico. La biopsia de grasa subcutánea abdominal en CA-TTR-h tiene positividad de $67 \%$ y solo de $14 \%$ en CA-TTR-wt. ${ }^{35}$

La biopsia de piel pareciera ser más sensible que la biopsia de grasa subcutánea en todos los tipos de amiloidosis, especialmente en CA-TTR (Figura 6) y en pacientes con polineuropatía y cardiopatía, la biopsia de nervio sural o de ganglios autonómicos es particularmente importante cuando existe sospecha de ATTR-h (Figura 6).

\section{Estudio Genético en CA-TTR}

El estudio genético es la herramienta que permite diferenciar CA-TTR-h de CA-TTR-wt. ${ }^{36}$ Existen más de 140 mutaciones del gen de la transtiretina, y se ha recomendado realizar estudio genético a todo paciente diagnosticado con CA-TTR, inclusive si no hay historia familiar de cardiopatía o polineuropatía. En la actualidad probablemente esto es clínicamente más relevante en pacientes jóvenes. La identificación de mutaciones puede tener relevancia clínica pronóstica y terapéutica. Las mutaciones que generan un compromiso fundamentalmente cardíaco son: Val122lle, Leu111Met y Ile68Leu (predominantemente en afro-americanos ${ }^{12}$, italianos o daneses, respectivamente). ${ }^{13-14}$. La polineuropatía idiopática sensitivo motora rápidamente progresiva asociada a hipertrofia cardíaca y/o a trastornos del ritmo cardíaco, debe orientar fuertemente al diagnóstico de ATTR-h. Las áreas endémicas para ATTR-h son Brasil, Portugal, Japón y Suecia.

\section{Diagnóstico diferencial y Algoritmo diagnóstico}

Las dos principales formas de CA, son la CA-TTR y la CA-AL. Esta última presenta ciertas características que la pueden distinguir de la CA-TTR.

La AL es la forma más común de amiloidosis sistémica, causada por una célula plasmática clonal. Se producen excesivamente cadenas livianas (kappa o lambda) de inmunoglobulinas, que se pliegan anormalmente depositándose en distintos tejidos. Los órganos más afectados son corazón, riñón, hígado y sistema nervioso (autonómico o periférico). A pesar de existir ciertos hallazgos, 


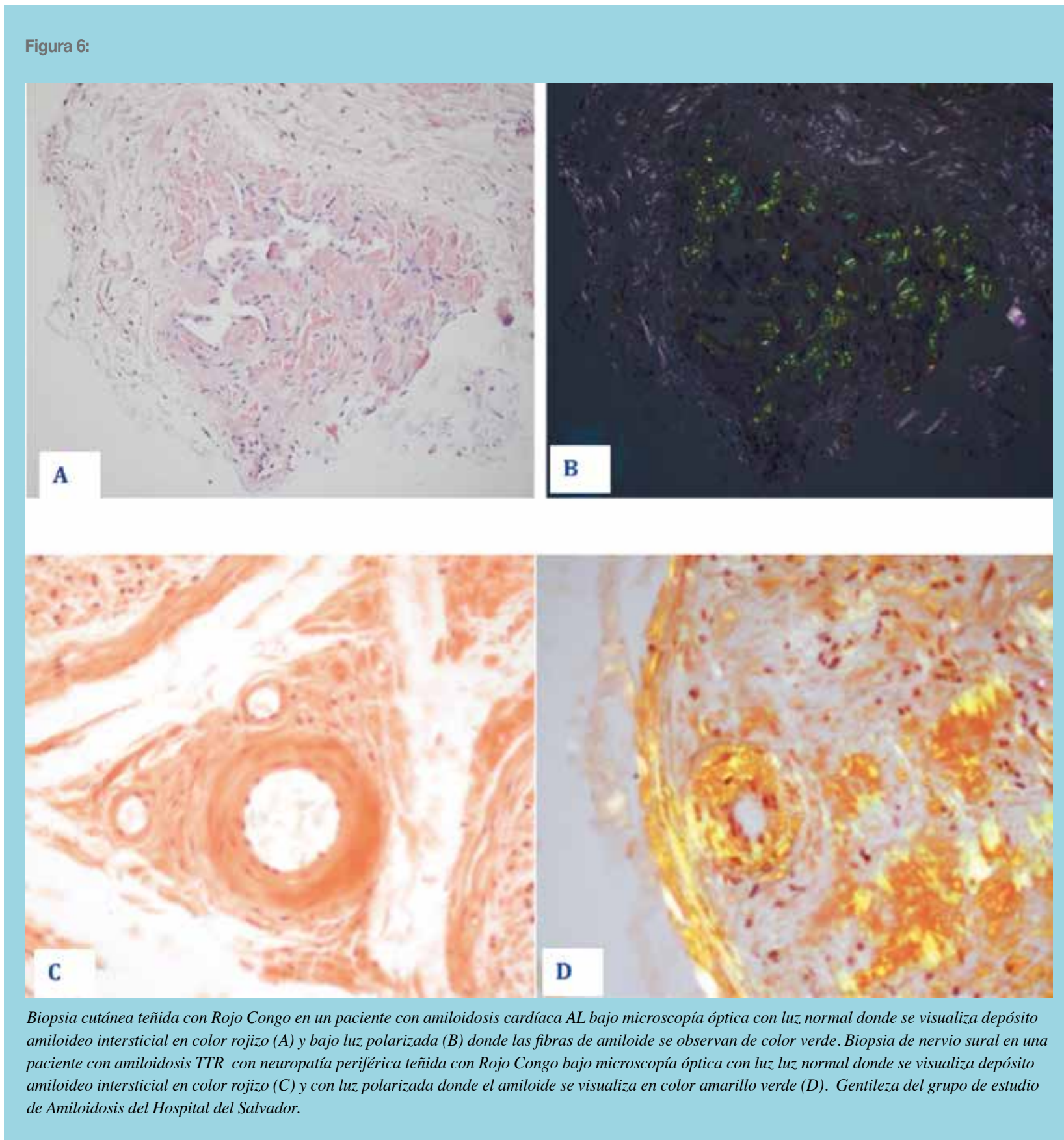

prácticamente patognomónicos (macroglosia y/o púrpura periorbital), estos son infrecuentes y en la gran mayoría de las ocasiones la manifestación clínica de esta enfermedad será falla cardíaca, síndrome nefrótico, neuropatía periférica o disfunción autonómica.

Para el diagnóstico de amiloidosis por cadenas livianas, es absolutamente necesario detectar la presencia de cadenas livianas monoclonales en suero y/u orina, y corroborar la infiltración de un órgano afectado con biopsia. ${ }^{37}$
El algoritmo diagnóstico propuesto (Figura 7), reúne las sugerencias de la asociación americana de cardiología. ${ }^{38}$ Debe aplicarse en todo paciente con alto índice de sospecha de CA-TTR, especialmente en presencia de elementos clínicos orientadores denominados banderas rojas o de alerta (Tabla 1). Muy sucintamente: ante la sospecha diagnóstica de CA primero detectar o descartar minuciosamente la presencia de cadenas livianas. $\mathrm{Si}$ no hay cadenas livianas y hay sospecha de CA por pre- 
Figura 7: Algoritmo para el diagnóstico de CA-TTR.

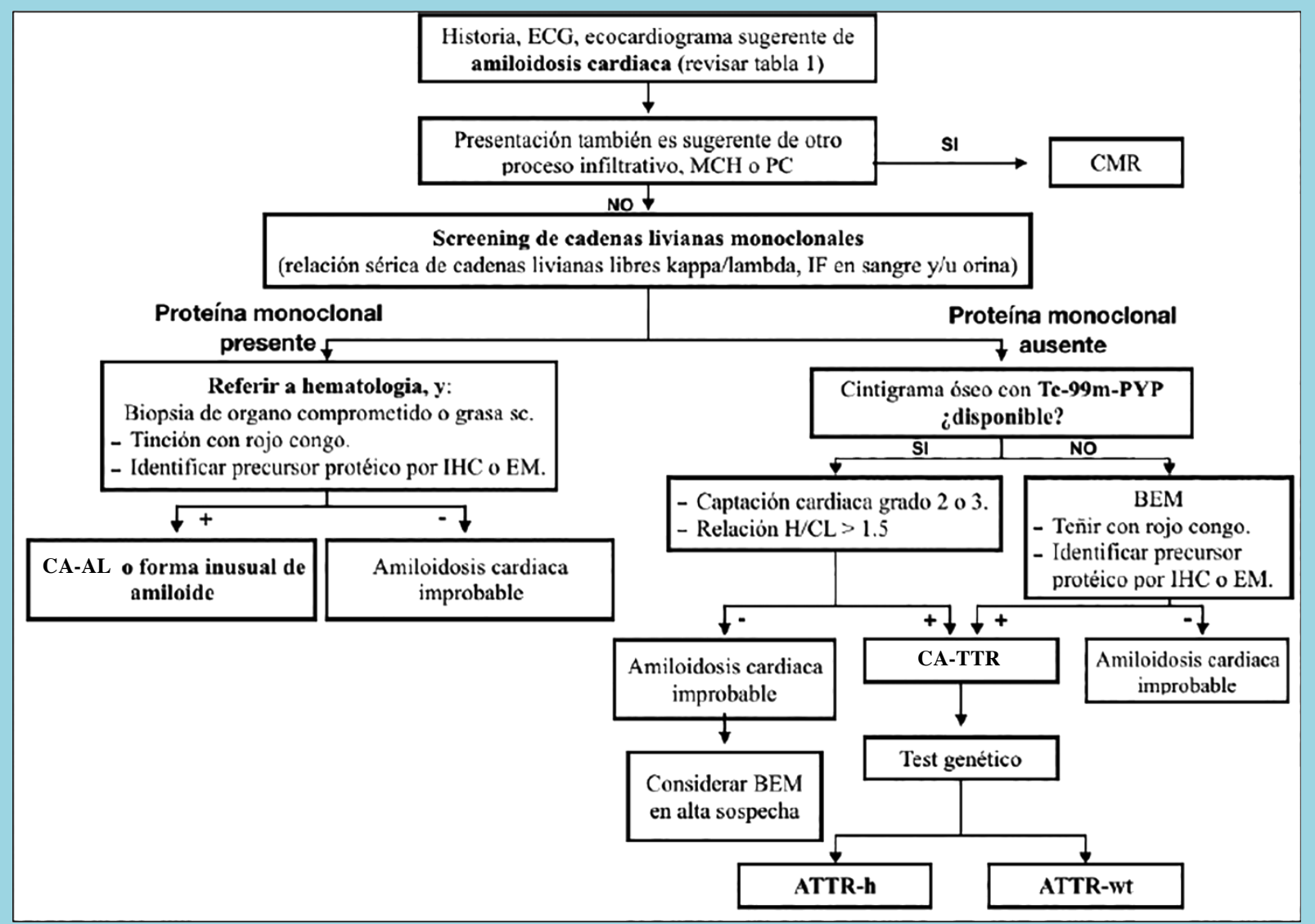

La resonancia magnética cardíaca (CMR) es útil en el diagnóstico diferencial de las miocardiopatías restrictivas, pericarditis constrictiva (PC) o en sospecha de miocarditis.

Se considera anormal una relación sérica de cadenas livianas libres kappallambda $<0.26$ o $>1.65$. MCH indica miocardiopatía hipertrófica; SC, subcutánea; IF, inmunofijación; IHC, inmunohistoquímica; EM, espectrometría de masas; AL-CM, cardiomiopatía por cadenas livianas; PYP, pirofosfato; BEM, biopsia endomiocárdica; H/CL, corazón/tórax contralateral; CA-TTR, cardiomiopatía por transtiretina; ATTR-h, amiloidosis por transtiretina hereditaria; ATTR-wt, amiloidosis por transtiretina natural.

sencia de banderas clínicas de alerta (Tabla 1), realizar cintigrafía con pirofosfato. Si está positiva estamos ante una CA-TTR.

\section{Tratamiento Se basa en 3 pilares: terapias modifi-} cadoras de la enfermedad, manejo de la falla cardíaca y de las arritmias.

Agentes modificadores de la enfermedad se consideran: el trasplante hepático-cardíaco y fármacos que inhiben la expresión genética de TTR y fármacos que estabilizan los tetrámeros.

El trasplante hepático se utiliza desde 1990 en ATTR-h y ha demostrado disminución sérica de TTR. Su utilización ha sido preferentemente en pacientes con polineuropatía (mutación V30M u otras mutaciones). En pacientes con cardiopatía y polineuropatía ATTR-h,
Tabla 1: Banderas de alerta o banderas rojas de amiloidosis cardíaca.

IC FEp en ausencia de hipertensión arterial

Estenosis aórtica de bajo flujo y bajo gradiente.

Intolerancia a antihipertensivos o a fármacos para el manejo de falla cardíaca. Antecedentes de síndrome del túnel carpiano bilateral, estenosis espinal lumbar o rotura espontánea del tendón del bíceps.

Elevación leve y persistente de troponinas.

Discrepancia entre el voltaje del QRS y el engrosamiento de la pared VI.

Fenotipo hipertrófico al ecocardiograma con patrón de bandera japonesa en imágenes del strain.

Realce tardío del gadolinio (LGE) de distribución subendocárdica o transmural en la resonancia nuclear magnética cardíaca. 
aparentemente el resultado es mejor con doble trasplante (hepático y cardíaco) que sólo hepático. Por otro lado, no hay claridad si existe beneficio con trasplante hepático en pacientes con mutación V122I, que comúnmente genera cardiomiopatía. ${ }^{39}$

Actualmente, el trasplante cardíaco por si solo no es parte del tratamiento en la CA-TTR, dada la recurrencia de la enfermedad, a diferencia de CA-AL., donde el trasplante de médula ósea y, según el grado de compromiso cardíaco, el trasplante cardíaco posterior tiene un rol más definido.

\section{Fármacos modificadores de la enfermedad.}

En estos últimos años se han desarrollado 2 terapias dirigidas a silenciar o estabilizar la TTR para mejorar la sobrevida de la CA-TTR con sobrevidas medias reportadas de 2.5 años en ATTR-h y de 3.6 años para ATTR-wt. ${ }^{40}$

Con el silenciamiento de TTR se evitan la síntesis de TTR a nivel hepático, al degradar su mRNA y reduciendo las concentraciones circulantes de TTR. Entre estos fármacos podemos encontrar patisiran ${ }^{41}$ e inotersen ${ }^{42}$, que han demostrado beneficios a nivel cardíaco en subgrupos de pacientes. ${ }^{43}$ Han sido aprobados por la FDA para el tratamiento de la ATTR-h asociada a neuropatía. Actualmente hay dos estudios en marcha en CA-TTR (APOLLO-B con Patisiran y HELIOS-B con Vutrisiran) evaluando la real eficacia clínica en esta patología. Los estabilizadores de TTR se unen al tetrámero de TTR y previenen su disgregación a dímeros, y finalmente a monómeros, previniendo el mal plegamiento y depósito de fibrillas de amiloide. Tafamidis, logra esta acción al unirse específicamente al sitio de unión de tiroxina. Su utilidad ha quedado evidenciada en el estudio ATTR-ACT, que demostró reducir la mortalidad global y las hospitalizaciones de causa cardiovascular a 30 meses, además de mejorar la capacidad funcional a los 6 meses. ${ }^{44}$ Este fármaco fue aprobado por la FDA para pacientes con CA-TTR para las formas hereditaria y natural (wild-type).

Muy recientemente las Guías Europeas de Insuficiencia Cardíaca 2021 recomiendan (1B) el uso de tafamidis en pacientes con CA-TTR hereditaria o wild type en CF I o II para reducir síntomas, hospitalizaciones y mortalidad 45 .

Por otro lado, está Diflunisal, un anti-inflamatorio no esteroidal (estabiliza TTR in vitro). No hay estudios clínicos controlados en ATTR-wt, y su eficacia y seguridad se ha evaluado solo en análisis retrospectivos.
Manejo de la falla cardíaca. No existen estudios específicos con respecto al tratamiento médico de esta cardiopatía en presencia de IC-FEp o de IC-FEr. La piedra angular en el manejo sintomático es mantener la euvolemia (restricción de sodio y uso de diuréticos con la precaución de no exacerbar la hipotensión arterial y de deteriorar la perfusión renal).

Los beta-bloqueadores deben usarse con precaución, ya que la única manera de mantener el gasto cardíaco en esta enfermedad es aumentando la frecuencia cardíaca. Se recomienda evitar los bloqueadores de canales de calcio no dihidropiridínicos dada su afinidad a las fibrillas de amiloide y el riesgo de bloqueo $\mathrm{AV}$ completo en casos reportados de CA-AL. Por otro lado, al avanzar la enfermedad, caerá el volumen de eyección y la presión arterial y el uso de inhibidores de angiotensina o antagonistas del receptor de angiotensina será mal tolerado. La digoxina está restringida, por el riesgo potencial de toxicidad secundario a su unión a fibrillas amiloides.

Manejo de las arritmias. Estos pacientes presentan disfunción atrial, por lo cual la indicación de anticoagulación en el caso de concomitancia con fibrilación o flutter auricular, es independiente del score Chads2-vasc. El antiarrítmico de elección es amiodarona para control del ritmo o de la frecuencia. El uso de marcapaso permanente se reserva para BAV de 2do grado Mobitz II y BAVC, independiente de la sintomatología. El implante de desfibrilador automático en prevención primaria no está bien establecido. Mientras que en prevención secundaria se debe guiar por las recomendaciones establecidas en las guías clínicas existentes europeas o americanas. ${ }^{46}$

\section{Conclusión:}

La CA-TTR es una enfermedad subdiagnosticada, heterogénea en su sintomatología. Para su diagnóstico requiere un alto índice de sospecha y de trabajo con otras especialidades (i.e. hematología, neurología). La cintigrafía ósea como examen no invasivo ha representado un cambio en el paradigma diagnóstico de esta enfermedad, evitando en la gran mayoría de los casos la necesidad de realizar biopsia endomiocárdica o de otros tejidos.

Por otro lado, los tratamientos farmacológicos emergentes en estos últimos años, han logrado aumentar la sobrevida de estos pacientes, por lo que resulta muy importante la implementación precoz de estas terapias en la evolución de la enfermedad. 


\section{Referencias}

1. GERTZ MA. Pathophysiology and treatment of cardiac amyloidosis. Nat. Rev. Cardiol. 2015;12:91-102.

2. GERTZ MA, BENSON MD, DYCK PJ. Diagnosis, prognosis, and therapy of transthyretin amyloidosis. J Am Coll Cardiol 2015;66:2451-2466.

3. RUBERG FL, GROGAN M, HANNA M. Transthyretin amyloid cardiomyopathy. J Am Coll Cardiol 2019;73:2872-91.

4. PEREIRA NL, GROGAN M, DEC GW. Spectrum of restrictive and infiltrative cardiomyopathies. J Am Coll Cardiol 2018;71:1130-48.

5. GONZALEZ-LOPEZ E, GALLEGO-DELGADO M, GUZZO-MERELLO G. Wild-type transthyretin amyloidosis as a cause of heart failure with preserved ejection fraction. Eur Heart J 2015;36:2585-94.

6. CASTANO A, NAROTSKY DL, HAMID N. Unveiling transthyretin cardiac amyloidosis and its predictors among elderly patients with severe aortic stenosis undergoing transcatheter aortic valve replacement. Eur Heart J 2017;38:2879-87.

7. DASARI A, HUNG I, GAN Z. Two distinct aggregation pathways in transthyretin misfolding and amyloid formation. Biochim Biophys acta proteins proteom 2019;1867(3):344-349.

8. MAURER MS, ELLIOTT P, COMENZO R. Addressing common questions encountered in the diagnosis and management of cardiac amyloidosis. Circulation 2017;135:1357-1377.

9. JACOBSON DR, ALEXANDER AA, TAGOE C. Prevalence of the amyloidogenic transthyretin (TTR) V122I allele in 14333 African-Americans. Amyloid 2015;22:171-174.

10. RAPEZZI C, QUARTA CC, OBICI L. Disease profile and differential diagnosis of hereditary transthyretin related amyloidosis with exclusively cardiac phenotype: an Italian perspective. Eur Heart J. 2013;34:520-528.

11. SVENDSEN IH, STEENSGAARD-HANSEN F, NORDVAG BY. A clinical, echocardiographic and genetic characterization of a Danish kindred with familial amyloid transthyretin methionine 111 linked cardiomyopathy. Eur Heart J. 1998;19:782-789.

12. NAKAGAWA M, SEKIJIMA Y, YAZAKI M. Carpal tunnel syndrome: a common initial symptom of systemic wild-type ATTR (ATTRwt) amyloidosis. Amyloid 2016;23:58-63.

13. GELLER HI, SINGH A, ALEXANDER KM. Association Be- tween Ruptured Distal Biceps Tendon and Wild-Type Transthyretin Cardiac Amyloidosis JAMA 2017;318:962-963.

14. WESTERMAK P, WESTERMARK GT, SUHR OB. Transthyretin-derived amyloidosis: probably a common cause of lumbar spinal stenosis. Ups J Med Sci 2014;119:223-8.

15. CONNORS LH, SAM F, SKINNER M. Heart failure resulting from age-related cardiac amyloid disease associated with wild-type transthyretin: a prospective, observational cohort study. Circulation 2016;133:282-90.

16. OERLEMANS MI, RUTTEN KH, MINNEMA MC. Cardiac amyloidosis: the need for early diagnosis. Neth heart $\mathbf{J}$. 2019;27:525-536.

17. CASTAÑO A, NAROTSKY DL, HAMID N. Unveiling transthyretin cardiac amyloidosis and its predictors among elderly patients with severe aortic stenosis undergoing transcatheter aortic valve replacement. Eur Heart J. 2017;38:2879-2887.

18. TERNACLE J, KRAPF L, MOHTY D. Aortic Stenosis and Cardiac Amyloidosis: JACC Review Topic of the Week. J Am Coll Cardiol. 2019;74:2638-2651.

19. GROGAN M, SCOTT CG, KYLE RA. Natural History of Wild-Type Transthyretin Cardiac Amyloidosis and Risk Stratification Using a Novel Staging System. J Am Coll Cardiol. 2016;68:1014-1020.

20. MURTAGH B, HAMMILL SC, GERTZ MA. Electrocardiographic findings in primary systemic amyloidosis and biopsy-proven cardiac involvement. Am J Cardiol. 2005;95:535-537.

21. RAPEZZI C, MERLINI G, QUARTA CC. Systemic cardiac amyloidoses: disease profiles and clinical courses of the 3 main types. Circulation. 2009;120:1203-1212.

22. DUNGU J, SATTIANAYAGAM PT, WHELAN CJ. The electrocardiographic features associated with cardiac amyloidosis of variant transthyretin isoleucine 122 type in Afro-Caribbean patients. Am Heart J. 2012;164:72-79.

23. MINTS YY, DOROS G, BERK JL. Features of atrial fibrillation in wild-type transthyretin cardiac amyloidosis: a systematic review and clinical experience. ESC Heart Fail. 2018;5:772-779.

24. KITAOKA, HIROAKI: JCS 2020 Guideline on Diagnosis and Treatment of Cardiac Amyloidosis. Circulation Journal 2020;25;84:1610-1671. 
25. PHELAN D, COLLIER P, THAVENDIRANATHAN P. Relative apical sparing of longitudinal strain using two-dimensional speckle-tracking echocardiography is both sensitive and specific for the diagnosis of cardiac amyloidosis. Heart. 2012;98:1442-1448

26. PAGOURELIAS, EFSTATHIOS D: Echo Parameters for Diferential Diagnosis in Cardiac Amyloidosis. A Head-to-Head Comparison of Deformation and Nondeformation Parameters. Circ Cardiovasc Imaging,2017,10.

27. PENUGONDA N. Cardiac MRI in infiltrative disorders: a concise review. Curr Cardiol Rev. 2010;6:134-136.

28. DUNGU JN, VALENCIA O, PINNEY JH. CMR-based differentiation of AL and ATTR cardiac amyloidosis. JACC Cardiovasc Imaging. 2014;7:133-142.

29. MESSROGHLI, DANIEL R: Clinical Recomedations for Cardiovascular Magnetic Resonance Mapping of T1,T2, T2* and Extarcellular Volume: A Consensus Stament by the Society for Cardiovascular Magnetic Resonance (SCMR) endorsed by the European Association for Cardiovascular Imaging (EACVI). Journal of Cardiovascular Magnetic Resonance (2017)19:75.

30. BAGGIANO, ANDREA: Noncontrast Magnetic Resonance for the Diagnosis of Cardiac Amyloidosis. JACC: Cardiovascular Imaging, Vol 13, $\mathrm{N}^{\circ} 1,2020$.

31. PERUGINI E, GUIDALOTTI PL, SALVIF. Noninvasive etiologic diagnosis of cardiac amyloidosis using 99mTc-3,3-diphosphono-1,2-propanodicarboxylic acid scintigraphy. J Am Coll Cardiol. 2005;46:1076-1084.

32. BOKHARI S, CASTAÑO A, POZNIAKOFF T. (99m) Tc-pyrophosphate scintigraphy for differentiating light-chain cardiac amyloidosis from the transthyretin-related familial and senile cardiac amyloidoses. Circ Cardiovasc Imaging. 2013;6:195-201

33. MAUER, MATHEW: Expert Consensus Recommendations for the Suspicion and Diagnosis of Transthyertine Cardiac Amyloidosis. Circ Heart Fail. 2019;12.

34. GILMORE JD, MAURER MS et al. Nonbiopsy Diagnosis of Cardiac Transthyretin Amyloidosis. Circulation. 2016;133:2404-12.

35. FINE NM, ARRUDA-OLSON AM, DISPENZIERI A. Yield of noncardiac biopsy for the diagnosis of transthyretin cardiac amyloidosis. Am J Cardiol. 2014;113:1723-1727.
36. MARTINEZ-NAHARRO A, BAKSI AJ, HAWKINS PN. Diagnostic imaging of cardiac amyloidosis. Nat Rev Cardiol. 2020;17:413-426.

37. DUBREY SW, CHA K, ANDERSON J, CHAMARTHI B, REISINGER J, SKINNER M, et al. The clinical features of immunoglobulin light-chain (AL) amyloidosis with heart involvement. QJM 1998; 91: 141-157.

38. KITTLESON MM, MAURER MS, AMBARDEKAR AV. Cardiac Amyloidosis: Evolving Diagnosis and Management: A Scientific Statement From the American Heart Association. Circulation. 2020;142:7-22.

39. EMDIN, MICHELE: Treatment of Cardiac Transthyretin Amyloidosis: an uptodate. European Heart Journal (2019) 40,3699-3706.

40. GROGAN M, SCOTT CG, KYLE RA. Natural History of Wild-Type Transthyretin Cardiac Amyloidosis and Risk Stratification Using a Novel Staging System. J Am Coll Cardiol. 2016;68:1014-1020.

41. ADAMS D, GONZALEZ-DUARTE A, O`RIORDAN WD. Patisiran, an RNAi Therapeutic, for Hereditary Transthyretin Amyloidosis. N Engl J Med. 2018;379:11-21.

42. BENSON MD, WADDINGTON-CRUZ M, BERK JL. Inotersen Treatment for Patients with Hereditary Transthyretin Amyloidosis. N Engl J Med. 2018;379:22-31.

43. SOLOMON SD, ADAMS D, KRISTEN A. Effects of Patisiran, an RNA Interference Therapeutic, on Cardiac Parameters in Patients With Hereditary Transthyretin-Mediated Amyloidosis. Circulation. 2019;139:431-443.

44. MAURER MS, SCHWARTZ JH, GUNDAPANENI B. Tafamidis Treatment for Patients with Transthyretin Amyloid Cardiomyopathy. N Engl J Med. 2018;379:1007-1016.

45. McDONAGH TA, METRA M, ADAMO M, GARDNER RS, BAUMBACH A, BOHM M , et al. 2021 ESC Guidelines for the diagnosis and treatment of acute and chronic heart failure. European Heart Journal 2021; 00, 1-128. doi:10.1093/ eurheartj/ehab368.

46. TOWBIN JA, MCKENNA WJ, ABRAMS DJ. 2019 HRS expert consensus statement on evaluation, risk stratification, and management of arrhythmogenic cardiomyopathy: executive summary. Heart Rhythm. 2019;16:373-407. 